\title{
A Novel Growth-Promoting Pathway Formed by GDNF- Overexpressing Schwann Cells Promotes Propriospinal Axonal Regeneration, Synapse Formation, and Partial Recovery of Function after Spinal Cord Injury
}

\author{
Ling-Xiao Deng, ${ }^{1,2}$ Ping Deng, ${ }^{2}$ Yiwen Ruan, ${ }^{1}$ Zao Cheng Xu, ${ }^{2}$ Nai-Kui Liu, ${ }^{1}$ Xuejun Wen, ${ }^{3}$ George M. Smith, ${ }^{4}$ \\ and Xiao-Ming $\mathrm{Xu}^{1,2}$ \\ ${ }^{1}$ Spinal Cord and Brain Injury Research Group, Stark Neurosciences Research Institute, and Department of Neurological Surgery, Goodman and Campbell \\ Brain and Spine, and ${ }^{2}$ Department of Anatomy and Cell Biology, Indiana University School of Medicine, Indianapolis, Indiana 46202, ${ }^{3}$ Department of \\ Chemical and Life Science Engineering, Virginia Commonwealth University, Richmond, Virginia 23284, and ${ }^{4}$ Shriners Hospitals Pediatric Research Center, \\ Temple University, School of Medicine, Philadelphia, Pennsylvania 19140
}

Descending propriospinal neurons (DPSN) are known to establish functional relays for supraspinal signals, and they display a greater growth response after injury than do the long projecting axons. However, their regenerative response is still deficient due to their failure to depart from growth supportive cellular transplants back into the host spinal cord, which contains numerous impediments to axon growth. Here we report the construction of a continuous growth-promoting pathway in adult rats, formed by grafted Schwann cells overexpressing glial cell line-derived neurotrophic factor (GDNF). We demonstrate that such a growth-promoting pathway, extending from the axonal cut ends to the site of innervation in the distal spinal cord, promoted regeneration of DPSN axons through and beyond the lesion gap of a spinal cord hemisection. Within the distal host spinal cord, regenerated DPSN axons formed synapses with host neurons leading to the restoration of action potentials and partial recovery of function.

\section{Introduction}

Descending propriospinal neurons (DPSNs) are important in mediating multiple spinal functions including reflex, posture, and locomotion (Conta and Stelzner, 2004). The greatest numbers of DPSNs are located in the medial part of Rexed's lamina VII and VIII (Matsushita et al., 1979; Conta and Stelzner, 2004; Reed et al., 2009). These neurons constitute an uninterrupted cell column, and their axons project bilaterally through the ventrolateral funiculi (Conta and Stelzner, 2004; Reed et al., 2009). DPSNs are known for their direct activation of motoneurons and interneurons as well as for their "functional relay" of supraspinal signals (Illert et al., 1977; Molenaar and Kuypers, 1978; Matsushita et al., 1979; Alstermark et al., 1991). Following spinal cord injury (SCI), supraspinal axons, such as those from the cor-

Received June 17, 2012; revised Jan. 23, 2013; accepted Jan. 30, 2013.

Author contributions: L.-X.D., Z.C.X., X.W., G.M.S., and X.-M.X. designed research; L.-X.D., P.D., Y.R., Z.C.X., N.-K.L., G.M.S., and X.-M.X. performed research;X.W. contributed unpublished reagents/analytic tools; L.-X.D., P.D., Y.R., Z.C.X., and X.-M.X. analyzed data; L.-X.D., G.M.S., and X.-M.X. wrote the paper.

This work was supported by the National Institutes of Health (NS036350, NS050243, NS052290, and NS059622), The Indiana Spinal Cord and Brain Injury Research Fund (ISCBIRF), and the Mari Hulman George Endowments. We thank Ms. Patti Raley for her critical reading of this manuscript.

The authors declare no competing financial interest.

Correspondence should be addressed to Dr. Xiao-Ming Xu, Spinal Cord and Brain Injury Research Group, Stark Neurosciences Research Institute, Indiana University School of Medicine, 950 West Walnut Street, R2-402, Indianapolis, IN 46202. E-mail: xu26@iupui.edu.

DOI:10.1523/JNEUROSCI.2973-12.2013

Copyright $\odot 2013$ the authors $\quad 0270-6474 / 13 / 335655-13 \$ 15.00 / 0$ ticospinal tract (CST), fail to regenerate through the lesion site (Sivasankaran et al., 2004; Liu et al., 2008). Therefore, regeneration of DPSN axons may provide an alternative pathway or functional relay that transmits supraspinal motor commands down to the spinal cord. The importance of such a repair strategy is further enhanced by the evidence that axons of DPSNs show greater growth potential after injury than those from long projecting supraspinal neurons (Richardson et al., 1980; David and Aguayo, 1981; Xu et al., 1997; Iannotti et al., 2003; Fenrich and Rose, 2009).

After SCI, axonal regeneration in a rostrocaudal orientation is essential for significant functional recovery. Because the formation of cavities inevitably occurs following SCI, an important strategy is to construct growth-permissive substrates, such as periphery nerves or cell-laden tissue engineered bridges, e.g., seeded with Schwann cells (SCs) (Richardson et al., 1980; David and Aguayo, 1981; Xu et al., 1997; Campos et al., 2004; Houle et al., 2006). This strategy supports successful regeneration of axons across the lesion gap to reach the distal graft-host interface. However, due to the presence of a growth-inhibitory environment associated with reactive astrocytes and CNS myelin on the host side of the graft-host interface, regenerating axons failed to depart from the bridging transplants back into the host spinal cord (Xu et al., 1997; Bunge, 2008; Xu and Onifer, 2009). Thus, strategies emphasizing additional treatments within the caudal host spinal cord, including providing attractive cues, are essential to reconstruct new functional circuits across the injury. 
Table 1. Summary of experimental design and animal groups

\begin{tabular}{|c|c|c|c|c|c|c|c|}
\hline \multirow[b]{3}{*}{ Group } & \multicolumn{2}{|c|}{$\begin{array}{l}\text { Construct a growth-promoting } \\
\text { pathway }\end{array}$} & \multirow{2}{*}{\multicolumn{4}{|c|}{ Assessments }} & \multirow[b]{3}{*}{ Total } \\
\hline & \multirow{2}{*}{$\begin{array}{l}\text { At the injury } \\
\text { gap }\end{array}$} & \multirow{2}{*}{$\begin{array}{l}\text { At the caudal } \\
\text { host cord }\end{array}$} & & & & & \\
\hline & & & Beh + BDA & $\mathrm{FG}$ & $\mathrm{EM}$ & E-phy & \\
\hline 1 & SCs-GDNF & DMEM & $n=5$ & $n=3$ & $n=1$ & $n=5$ & $n=14$ \\
\hline 2 & SCs-GDNF & SCs-GFP & $n=7$ & $n=3$ & $n=1$ & $n=5$ & $n=16$ \\
\hline 3 & SCs-GDNF & SCs-GDNF & $n=7$ & $n=4$ & $n=4$ & $n=6$ & $n=21$ \\
\hline
\end{tabular}

BDA, biotinylated-dextran amine; Beh, behavior; EM, electron microscopy; FG, Fluoro-Gold; E-phy, electrophysiological recording.

Glial cell line-derived neurotrophic factor (GDNF) and its receptors are widely expressed in the developing and adult CNS. GDNF has a broad effect on recovery after injury, including neuronal survival, protection, axonal regeneration, remyelination, and synaptic formation (Perrelet et al., 2002; Iannotti et al., 2003; Ledda et al., 2007; Zhang et al., 2009). In the present study, we sought to determine the efficacy of a new combinatory strategy by reconstructing a continuous growth-promoting pathway, formed by grafted SCs overexpressing GDNF (SCs-GDNF), which not only bridges the lesion gap but also extends into the caudal host spinal cord to enhance axonal growth through and beyond an SCI. We hypothesized that successful regeneration and re-innervation of the DPSNs establishes a functional relay that constructs new descending spinal circuits leading to recovery of motor function following SCI.

\section{Materials and Methods}

Generation of purified SCs. SCs were purified as described previously (Morrissey et al., 1991; Xu et al., 1995). Purified SCs (purity >98\%) at the third or fourth passage were collected for in vitro infection and transplantation.

Transduction of SC in vitro. SCs were pretreated with $4-6 \mu \mathrm{g} / \mathrm{ml}$ polybrene (Sigma-Aldrich) for 30-60 min, infected by lentiviruses expressing either green fluorescent protein (SCs-GFP) or GDNF (SCs-GDNF), or both (SCs-GFP/GDNF; for demonstrating survival of grafted SCs in the caudal host spinal cord) at multiplicity of infection (MOI) of 4 , resulting in about 50\% infection of cells (Abdellatif et al., 2006; Deng et al., 2011). ELISA result showed that SCs, infected with lenti-GDNF $(\mathrm{MOI}=4)$, significantly increased GDNF production $(65.05 \pm 7.89$ $\mathrm{pg} / \mathrm{ml} / 10^{6}$ cell $/ 24 \mathrm{~h}$ ), which was $\approx 60$-fold higher than that produced by normal SCs or SCs infected with lenti-GFP $(p<0.001)$.

Seeding SCs into mini-guidance channels. SCs-GDNF was seeded at a final density of $120 \times 10^{6}$ cells $/ \mathrm{ml}$ into semipermeable polyacrylonitrile/ polyvinyl chloride copolymer guidance channels with an outer diameter of $1.25 \mathrm{~mm}$ (provided by Dr. Xuejun Wen, Virginia Commonwealth University, Richmond, VA) according to a previously described method (Xu et al., 1999).

Animal groups. A total of 51 adult female Sprague Dawley rats (180$200 \mathrm{~g}$, Harlan) were randomly divided into three groups (Table 1). All animals received a right-sided spinal cord hemisection at $\mathrm{T}-11$ followed by implantation of a $3 \mathrm{~mm}$ long SCs-GDNF-seeded guidance channel. In the distal host spinal cord, a growth-promoting pathway was constructed by injecting either DMEM $(n=14)$, SCs-GFP $(n=16)$, or SCs-GDNF $(n=21)$, into the distal host spinal cord (described below). Furthermore, to display the relationship between grafted SCs and regenerated axons in the caudal spinal cord, we had an extra group of rats $(n=7)$ that received grafts of SCs that had been transfected with lentivirus-GFP and lentivirus-GDNF sequentially. To keep all researchers blinded to the treatment groups during behavioral assessments, surgeries, and electrophysiological recordings, we established a standard practice of coding all animals with numbers that are randomized and not reflective of treatment groups. Information about the type of treatment is separated from the coded numbers immediately following treatment and is not present during these procedures. All animal surgical procedures and postoperative care were performed in accordance with the Guide for the Care and
Use of Laboratory Animals (National Research Council) and the Guidelines and Policies for Rodent Survival Surgery provided by the Animal Care Committee of Indiana University.

Spinal cord hemisection, transplantation of SC-seeded guidance channels, and caudal injection of SCs. The procedures for spinal cord hemisection and mini-guidance channel implantation were described in previous publications (Xu et al., 1999; Deng et al., 2011). The spinal cord hemisection was performed at the T-11 level. DMEM, SCs-GFP, SCs-GDNF, or SCs-GFP/GDNF was injected into the caudal host spinal cord on the graft side at the time of channel implantation. The injections were made at 0.5 and $1 \mathrm{~mm}$ caudal to the edge of grafted channel and at $0.5 \mathrm{~mm}$ lateral to the midline and $1 \mathrm{~mm}$ ventral to the dorsal surface of the spinal cord. At each injection point, $1 \mu \mathrm{l}$ of DMEM or SCs (-GFP or -GDNF; density: $\left.1 \times 10^{5} \mathrm{cell} / \mu \mathrm{l}\right)$ in DMEM was injected.

Behavior assessments. Basso, Beattie, and Bresnahan (BBB) locomotor rating scale was performed every 2 weeks after SCI for up to 6 weeks (Basso et al., 1995) (see Fig. 1). The contralateral intact spinal cord was transected at the seventh week and BBB was re-assessed at the eighth week to evaluate functional recovery when only the regenerated transplant connected to the rostral and caudal spinal cords. Footprint analysis was conducted at the sixth week after transplantation according to our existing protocol (Titsworth et al., 2007).

Anterograde and retrograde tracing. On the seventh week after spinal cord hemisection and transplantation, bilateral and stereotaxical injections of biotinylated-dextran amine (BDA; 10\%, $1 \mu \mathrm{l} /$ site; Molecular Probes) were made into the intermediate gray matter of the T9-T10 cord at distances of 3-6 $\mathrm{mm}$ rostral to the graft (for BDA, 1 injection/site $/ \mathrm{mm}$ ) according to a previously published work (Bamber et al., 2001). For the retrograde tracing, animals received stereotaxical injections of $0.5 \mu \mathrm{l}$ of Fluoro-Gold (FG; $2 \%$; Fluorochrome) into the spinal cord (from the midline $0.3 \mathrm{~mm}$; from the dorsal surface of the spinal cord $1.2 \mathrm{~mm}$ ) on the injury side at $1.5 \mathrm{~mm}$ distal to the guidance channel. Before the injection, the left intact hemicord was completely transected to prevent labeling of any axons coursing around the graft (Xu et al., 1999). The number of animals that received anterograde or retrograde tracings can be found in Table 1 .

Electrophysiological recording. To determine whether regenerating axons form functional synaptic contacts, extracellular recordings of field potentials were performed in vivo stereotaxically. Electrical stimuli were delivered with a bipolar stimulating electrode inserted into the intermediate gray region (where DPST neurons are originated) at 3-6 $\mathrm{mm}$ rostral to the lesion. The stimulating electrode was made from 000 stainless steel insect pins, insulated except for within $1 \mathrm{~mm}$ of the tops. Stimuli were constant current pulses of $0.1 \mathrm{~ms}$ duration ranging from $0.1-3 \mathrm{~mA}$. The recording electrode was pulled from glass capillaries with a tip resistance of $\sim 5 \mathrm{~m} \Omega$ when filled with $2 \mathrm{M} \mathrm{NaCl}$. Field potentials were evoked at 1,2, and 4 times the threshold stimulating intensity. Field potential was recorded from the ipsilateral spinal cord caudal to the SC bridge transplant (where DPST axons terminate) under several conditions as follows: (1) the presence of an intact contralateral hemicord, (2) transection of the contralateral hemicord to remove descending input through it, and (3) transection of the ipsilateral hemicord at the rostral grafthost interface to remove descending input through the graft. Recordings were performed with an Axoclamp 2B amplifier (Molecular Devices). Signals were digitized using a data-acquisition program and stored for off-line analysis. To quantify stimulus-induced voltage responses, we measured the amplitude of field potential as the difference between the baseline voltage (average voltage of $300 \mathrm{~ms}$ after response) and peak voltage of responses (occurred at about $50 \mathrm{~ms}$ after stimulus). For each rat, the maximal amplitude was used for comparison between different treatments.

Electron microscopy. Briefly, rats were perfused with $2 \%$ paraformaldehyde and $1 \%$ glutaraldehyde followed by $5 \%$ sucrose in phosphate buffer. Sections underwent a freeze-thaw procedure, incubated in avidinbiotin-peroxidase complex (1:50; Vector Laboratories) containing 2\% normal goat serum in PBS, visualized with nickel-DAB/ $\mathrm{H}_{2} \mathrm{O}_{2}$, and followed by conventional processes for transmission electron microscopy (Yan et al., 1999).

Immunohistochemistry. Polyclonal rabbit anti-glial fibrillary acidic protein (GFAP) antibody (1:100, Millipore Bioscience Research Re- 


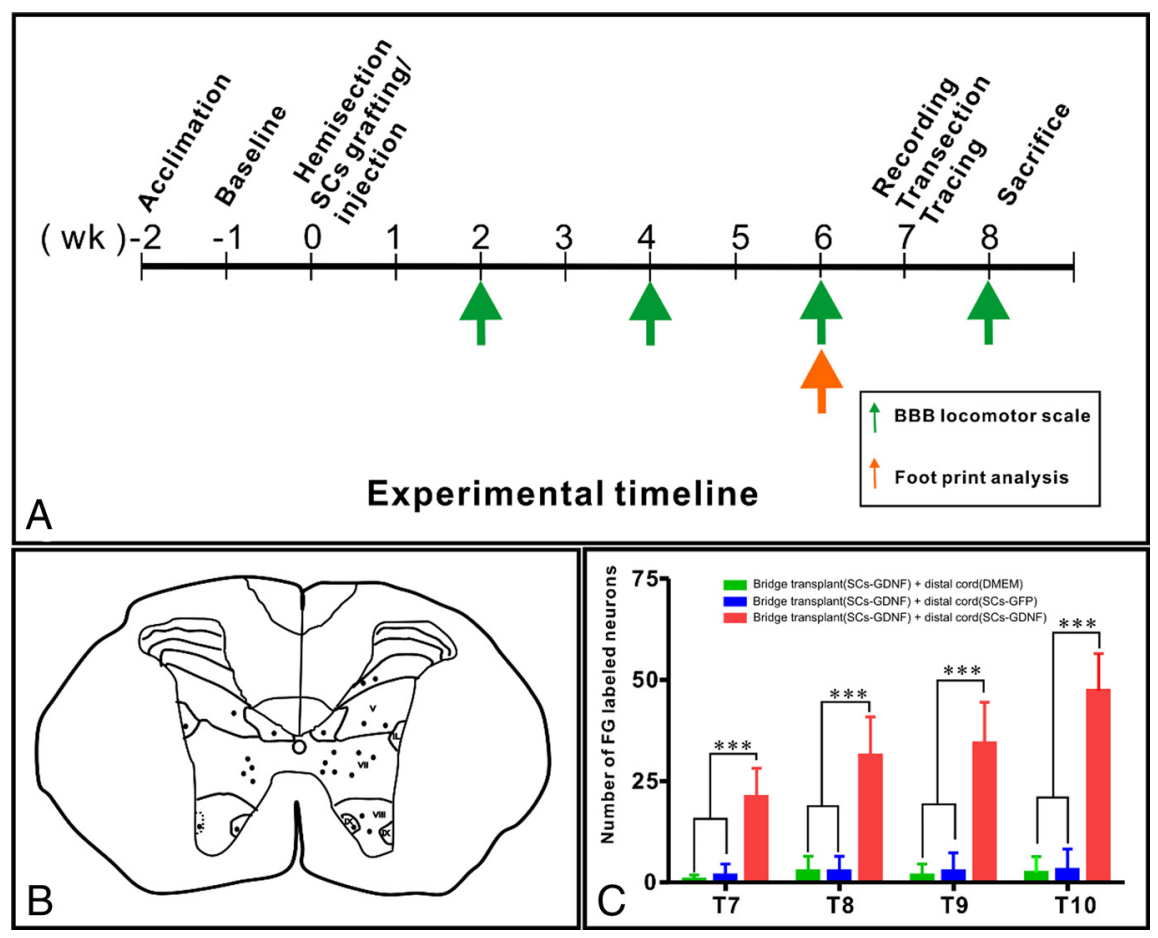

Figure 1. The majority of neurons whose axons extended into the distal host spinal cord originated from descending propriospinal neurons. $A$, Experimental time line. $\boldsymbol{B}$, Schematic drawing displayed the distribution of FG retrogradely labeled propriospinal neurons. Most labeled neurons were located in the intermediate gray matter and medial portion of the ventral horn (laminae V-VIII) between the T7-T10 cord segments. C, Bar graph shows that significantly more FG-labeled neurons were found in the group when SCS-GDNF was injected into the caudal spinal cord to form a continuous growth-promoting pathway compared with groups with injections of either SCs-GFP or DMEM at these spinal levels $\left({ }^{* * *} p<0.001\right)$.

agents), anti-microtubule-associated protein 2 (MAP2) antibody (1:100; Sigma), anti-P0 antibody (1:100, Millipore), and monoclonal mouse anti-synaptophysin antibody (1:1000; Millipore Bioscience Research Reagents) were used to identify astrocytes, neurons/dendrites, myelin, and presynaptic components, respectively, according to an existing protocol (Deng et al., 2011).

Quantification of BDA-labeled axons and FG-labeled propriospinal neurons. For quantification of BDA-labeled axons, three sets of serial BDAstained horizontal sections cut at a thickness of $20 \mu \mathrm{m}$ and spaced at 100 $\mu \mathrm{m}$ between adjacent sections through the guidance channel and tissue cable were used for analysis. We defined the distal host-graft boundary (marked by GFAP staining) as a starting point " 0 " to measure the distances of regenerated axons appearing in the host caudal spinal cord at every $400 \mu \mathrm{m}$ interval. The number of BDA-labeled axons on each section was counted and summed. For animals receiving FG retrograde tracer injections, the rostral spinal cord segments were sectioned transversely and serially at $40 \mu \mathrm{m}$. The number of FG-labeled cells was counted according to an existing protocol (Iannotti et al., 2003).

Animal exclusion criteria. To avoid identifying intact axons as regenerating axons, we performed a second surgery to completely transect the remaining nonlesioned spinal cord contralateral to the graft. We then used the following highly conservative criteria to evaluate the completeness of the second transection and the possible mislabeling of nonregenerated axons. A rat was excluded if (1) examination of every 20 - $\mu \mathrm{m}$-thick section, stained for GFAP, indicated an incomplete interruption of GFAP-labeled astrocytes in the contralateral cord after its transection or (2) if BDA-labeled axons were found to bypass the grafted guidance channel through the contralateral hemicord with an incomplete lesion (see Fig. 3). For animals receiving FG tracer injections, the same criteria applied for an incomplete transection. Additionally, in cases when FG was found to spread into grafted channel, it was excluded. In cases when the animal did not survive the second surgery, all their behavior scores were excluded from analysis. On the basis of these criteria, rats summarized in Table 1 were the final numbers included in the data collection.
Statistical analysis. Data are presented as mean \pm SD. Two-way ANOVA was used to determine statistical significance with Tukey's post hoc analysis. All statistical values were calculated using GraphPad Prism 5.0 software (GraphPad), with a $p$ value $<0.05$ considered statistically significant.

\section{Results \\ Propriospinal axons regenerate back into the distal host spinal cord}

We first determined the origin of neurons whose axons regenerated through and beyond an SCs-GDNF growth-promoting pathway. Retrograde tracer (FG) was injected into the host spinal cord $1.5 \mathrm{~mm}$ distal to the graft to label neurons from axons regenerating into or beyond the injection site caudal to the graft. Our results showed FG-labeled neurons rostral to the injury/graft mainly in the group that received SCs-GDNF within the transplant and caudal to it (Fig. 1). Most labeled neurons whose axons had regenerated at or beyond the injection site originated from propriospinal neurons located in lamina VII and the medial portion of lamina VIII, although some were also found in laminae III-VI and X. The labeled neurons were distributed bilaterally in the spinal cord; although, more labeled cells were found on the ipsilateral side. Greater numbers of FG-labeled neurons were found closer to the injury/graft site (Fig. 1). The highest number of labeled neurons was found between T8-T10 spinal cord levels. FG-labeled neurons were not found in supraspinal regions. In a pilot study, we determined that, in normal rats, FG can be transported from the injection site at the lumbar cord to the rostral spinal cord, red nucleus, and motor cortex at 1 week postinjection (data now shown) indicating that 1 week is sufficient for FG to be transported from the injection site to the brainstem and motor cortex. Thus, current data indicate that neurons whose axons regenerated through and beyond the SCs-GDNF grafts originated mainly from propriospinal neurons in close proximity to the injured segment.

To trace the course of regenerated propriospinal axons directly through and beyond the bridge transplant, we injected an anterograde tracer, $\mathrm{BDA}(2 \%)$ into the intermediate gray matter of the T9-T10 cord at distances 3-6 mm rostral to the graft. Considering the possibility of bilateral projections or sprouting of injured propriospinal axons across the midline, it is necessary to transect the contralateral intact hemicord before evaluating axon regeneration caudal to the transplant. According to strict criteria that we set up (see Materials and Methods, Animal exclusion criteria), a qualified rat should display the histological evidence in serial horizontal sections of the spinal cord that (1) the lesion, outlined by GFAP-immunoreactivity (IR), should be complete (Figs. $2 A, 3 A-C$ ), and (2) BDA-labeled axons did not bypass the transected contralateral hemicord (Figs. 2A, $4 B-E$ ). Two rats in the DMEM group, one rat in the SCs-GFP group, and one rat in the SCs-GDNF group were considered to have an incomplete lesion on the contralateral hemicord; one rat in the DMEM group and SC-GFP group did not survive after the second cord transection. These rats were all 


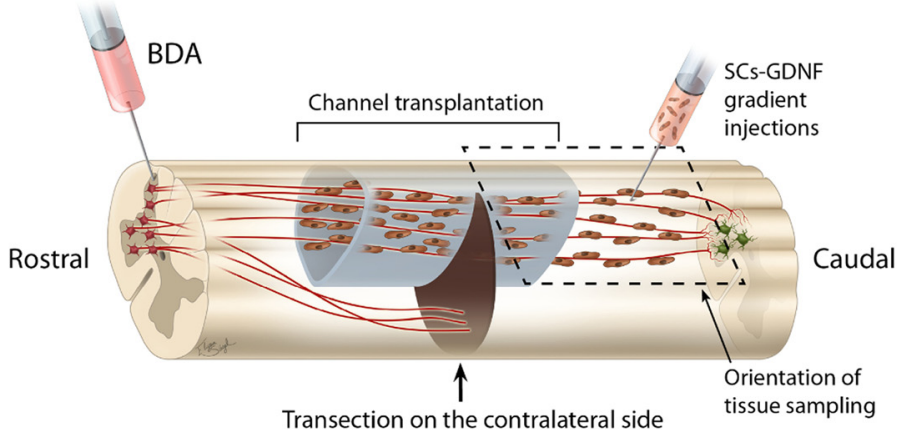

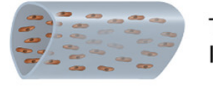

Transplanted channel loaded with SCs

\section{SCs-GDNF gradient} injections
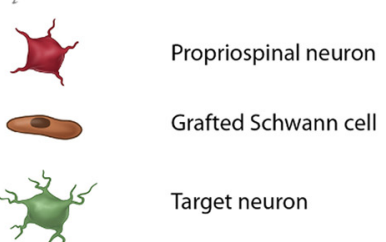
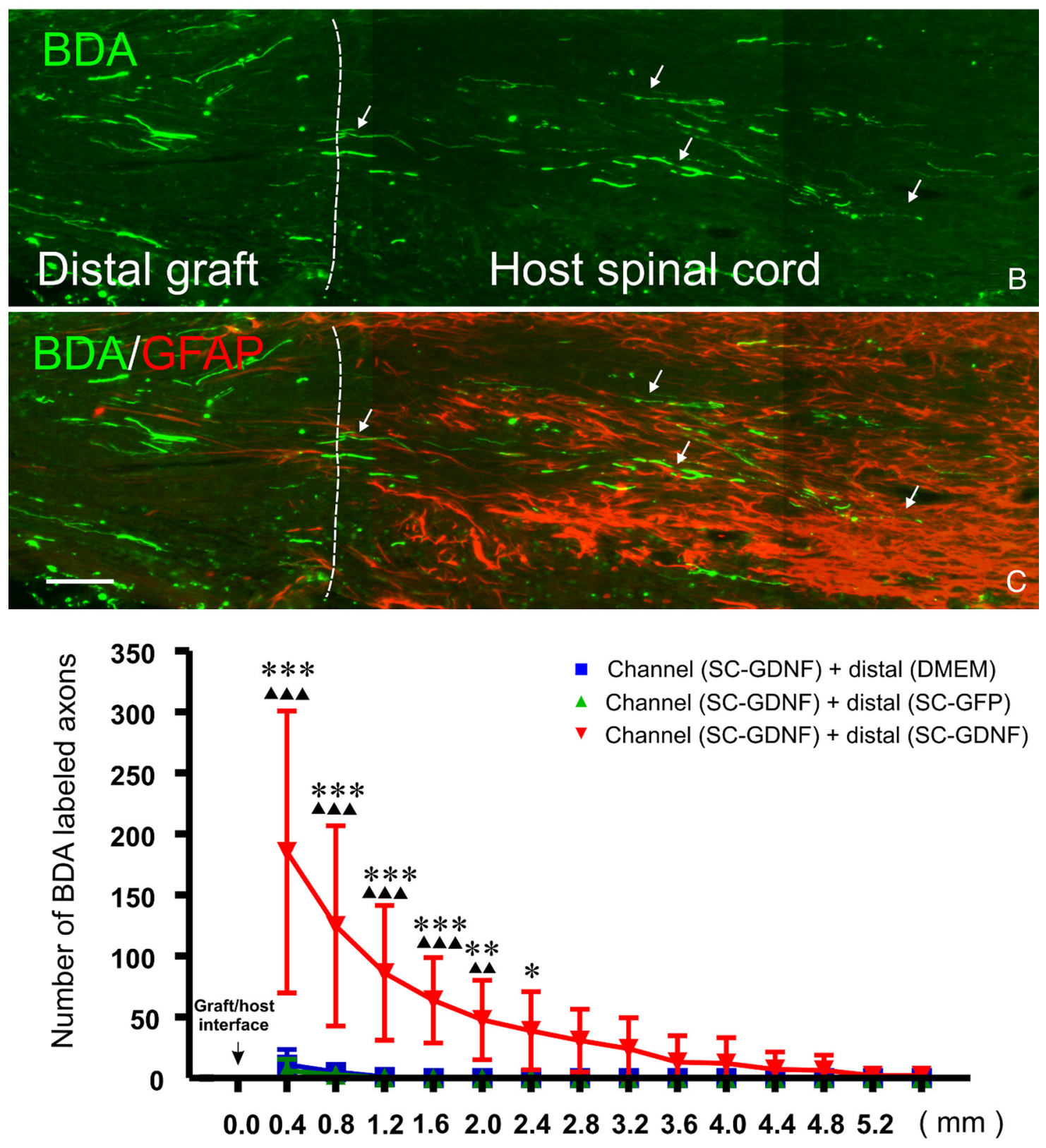

Distance to the graft-host interface

Figure 2. Descending propriospinal axons regenerated across the caudal graft-host interface and grew back into the distal host spinal cord. $\boldsymbol{A}$, Schematic drawing shows the experimental strategy and how tissue was sampled. Note that labeled axons descending down to the contralateral hemicord were stopped at the rostral edge of a complete transection of the hemicord made right before the tracer injection. $\boldsymbol{B}, 0 \mathrm{n}$ the ipsilateral side of the bridge transplantation, BDA (green) anterogradely labeled propriospinal axons (white arrows) were found to (Figure legend continues.) 

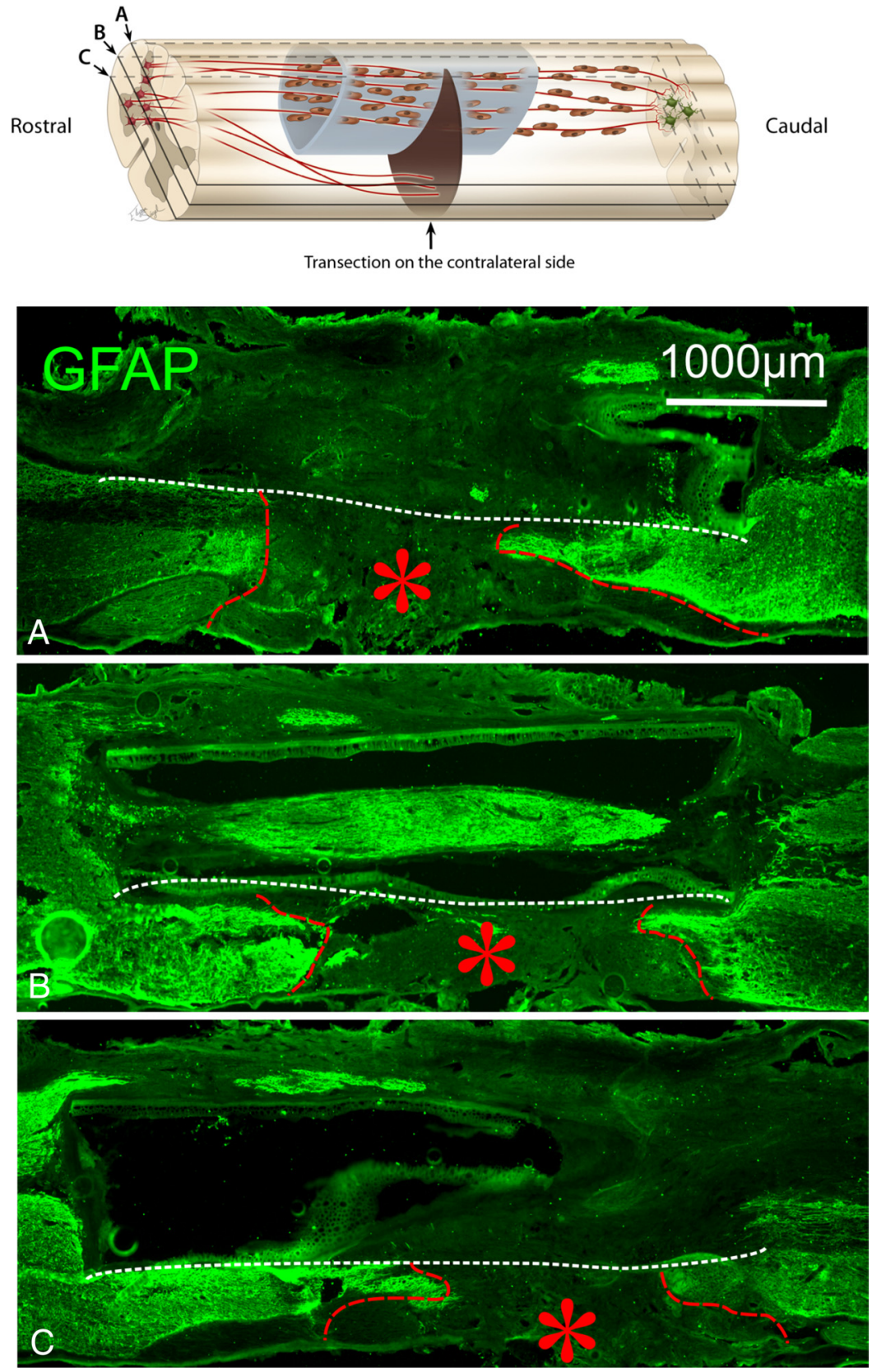

Figure 3. Evidence for complete transection of the hemicord contralateral to the bridge transplantation. Schematic drawing illustrates the contralateral hemisection and how samples were taken. Three horizontal sections represent the dorsal $(\boldsymbol{A})$, middle $(\boldsymbol{B})$, and ventral ( $\boldsymbol{C}$ ) portions of the spinal cord. GFAP staining (green) outlines rostral and caudal boundaries of the transection (red dashed lines). Between the two boundaries, a clear complete transection of the contralateral hemicord $\left({ }^{*}\right)$ was seen. Dashed white lines outline the medial wall of the guidance channel.

excluded from further analysis. In control groups, in which either SCs-GFP or DMEM was injected into the distal host tissue, outgrowth was sparse and limited to within $400-600 \mu \mathrm{m}$ of the grafthost interface characterized by GFAP staining (SCs-GFP group:

$\leftarrow$

(Figure legend continued.) penetrate through the distal graft-host interface (white dashed line) and to elongate within the distal host spinal cord only in the group injected with SCS-GDNF into the caudal host tissue. $C$, The distal graft-host interface was demarcated by GFAP-labeled astrocytes (red). D, Quantification of regenerated axonal numbers at different distances from the graft-host interface among three treatment groups. $\left({ }^{* * *} p<0.001,{ }^{* *} p<0.01,{ }^{*} p<\right.$ 0.05, compared with the distal SC-DMEM group; ${ }^{\mathbf{\Lambda \Lambda}} \mathbf{\Delta}_{p}<0.001, \boldsymbol{\Delta \Lambda}_{p}<0.01$, compared with the distal SC-GFP group). Scale bars: (in C) B, C, $100 \mu \mathrm{m}$.
Caudal

$2.5 \pm 4.342, n=7$; DMEM group: $3.25 \pm$ $4.86, n=5$, at $0.4 \mathrm{~mm}$ distal to the grafthost interface). While in the SCs-GDNF group, a large number of regenerated axons penetrated though the interface $(195.1 \pm$ $113.4, n=7$, at $0.4 \mathrm{~mm}$ distal to the interface). The longest axons that extended into the caudal cord were found to be at $5.0 \mathrm{~mm}$ from the distal interface (Fig. $2 A-D$ ).

\section{Propriospinal axons regenerated} through the bridge transplant to extend into the distal host spinal cord

To avoid false positive results due to an incomplete lesion of the spinal cord allowing spared axons to bypass the lesion to enter the caudal cord, it is necessary to provide histological evidence of completeness of the lesion in serial sections. In all cases, we not only carefully judged the lesion gap on the contralateral side by visual observation during the relesioning surgery, but also by histological and immunohistochemical assessments on serial sections (Figs. 3, 4). In Figure 3, representative three sequential sections from dorsal to ventral were shown. GFAP staining outlines a clear complete transection of the contralateral hemicord between the rostral and caudal astrogliotic boundaries (Fig. $3 A-C$ ). The completeness of the lesion was further confirmed by BDA axonal tracing (Fig. 4). In both horizontal (Fig. $4 B$ ) and cross (Fig. $4 C-E$ ) sections in Figure 4, many BDAlabeled axons appeared in the rostral contralateral hemicord; however, they were all stopped at the rostral end of the lesion gap after the complete transection of the contralateral hemicord. In the distal portion of the transection, no BDA-labeled axons were found to escape from the transected site. Thus, BDA-labeled axons found in the caudal host spinal cord were exclusively derived from those regenerated through the bridge transplant.

Regenerated propriospinal axons form synapses on host spinal cord neurons

To determine whether regenerated propriospinal axons form new synapses on target neurons in the distal host spinal cord, colocalization of BDA-labeled axons with a presynaptic marker, synaptophysin, and a neuronal marker, MAP2, was performed. At low magnification, BDA-labeled regenerated propriospinal axons were clearly seen to penetrate through the graft-host interface (demarcated by the opening of the implanted channel wall) and to elongate for considerable distances within the distal host spinal cord (Fig. 5A). At high magnification of boxed areas in Figure $5 A$, colocalization was observed with a presynaptic vesicle marker synaptophysin (green) on BDA-labeled propriospinal axons (red) and local neuron/dendrites (blue; MAP2 ${ }^{+}$; Fig. $5 B, C$ ). Since regenerated axons innervated both the ventral and intermediate zones (Fig. $5 D-F)$, the dendrites that these axons contacted are likely derived 

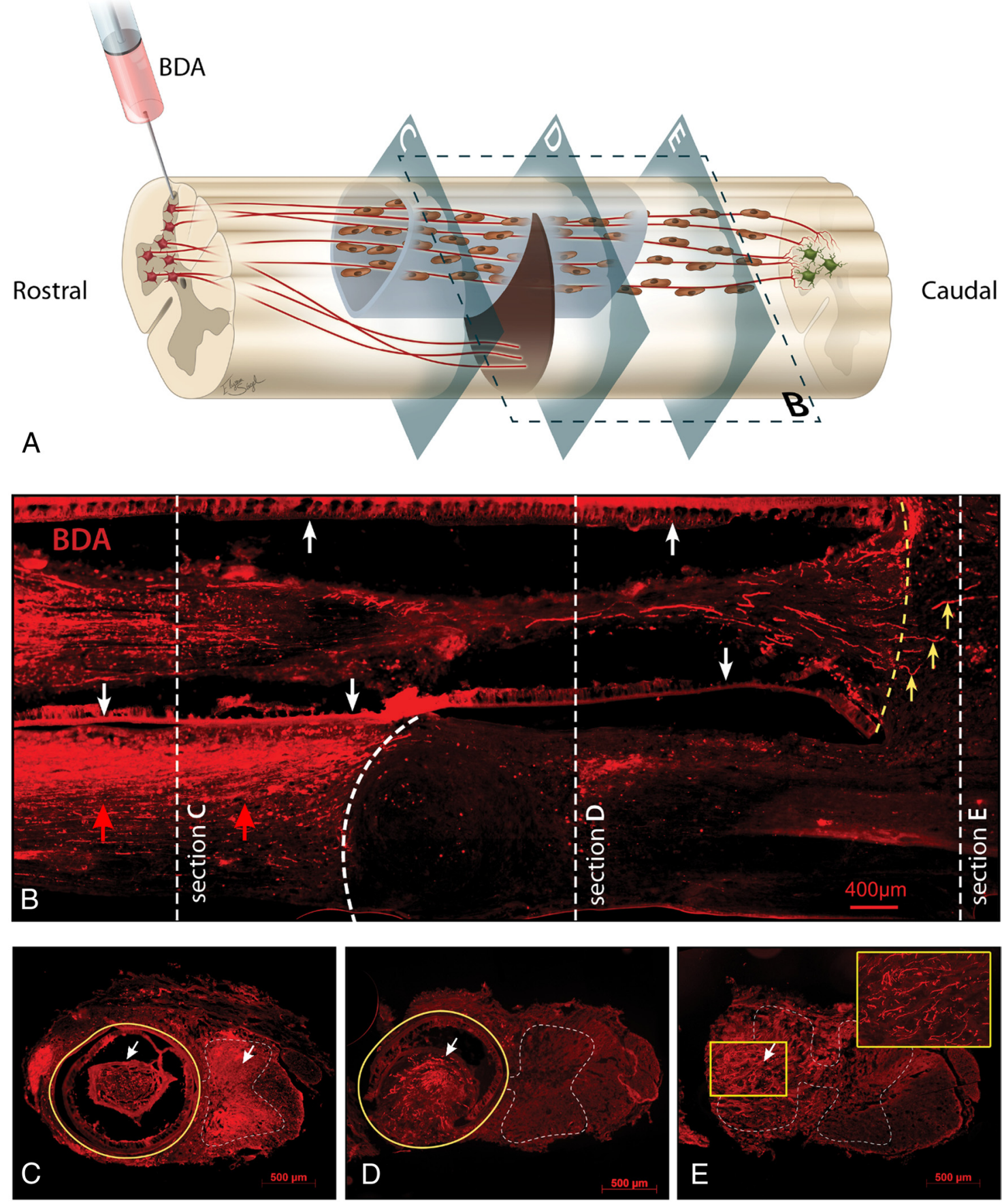

Figure 4. Only BDA-labeled axons that regenerated through the bridge transplant re-enter the distal host spinal cord. $A$, Schematic drawing illustrates that BDA-labeled axons regenerate through and beyond a bridge transplant. In the contralateral hemicord, however, BDA-labeled axons are completely stopped after the transection of spared hemicord. $\boldsymbol{B}$, A representative horizontal section shows that BDA-labeled axons grew through a regenerative tissue cable, penetrated the distal graft-host interface (yellow curved line), and re-entered the host spinal cord (yellow arrows). On the contralateral side, however, the labeled axons (red arrows) completely stopped at the site of hemicord transection (white curved line). $C-E$, representative cross sections taken from corresponding levels of the graft and adjacent cord tissue shown in $\boldsymbol{B}$ (white straightlines). C, Within the graftrostral to the contralateral transection, BDA-labeled axons (white arrows) were seen both within the graft and the contralateral hemicord. $\boldsymbol{D}$, Within the graft caudal to the contralateral transection, BDA-labeled axons were found only within the graft (white arrow). $\boldsymbol{E}$, In the host spinal cord caudal to the graft-host interface, BDA-labeled regenerated axons appeared only in the spinal cord ipsilateral to the graft. High magnification of the boxed area in $\boldsymbol{E}$ shows details of $B D A$-labeled regenerated axons in the distal host spinal cord. Arrows in $B$ indicate the guidance channel wall. Yellow circles in $\mathbf{C}$ and $\boldsymbol{D}$ outline the guidance channel wall in cross sections. White dashed lines in $\mathbf{C}-\boldsymbol{E}$ outline the gray matter areas. Scale bars: $\boldsymbol{B}, 400 \mu \mathrm{m} ; \boldsymbol{C}-\boldsymbol{E}, 500 \mu \mathrm{m}$.

from local interneurons or motoneurons. To obtain more solid evidences for synapse formation, we examined the samples with BDA immunoelectron microscopy (EM). We found that regenerated axons form presynaptic terminals with numerous synaptic vesicles, which were electron dense after immunolabeling for BDA reacted with streptavidin-horseradish peroxidase (Fig. 5C, lower right insert).

\section{Grafted SCs survived, facilitated axonal regeneration, and} formed new myelin on regenerated axons in the distal host spinal cord

To determine whether grafted SCs survived and formed a growth-promoting pathway that facilitated axonal regeneration in the distal host spinal cord, we transfected SCs with lentivirusGFP and lentivirus-GDNF sequentially (SCs-GFP/GDNF) and 

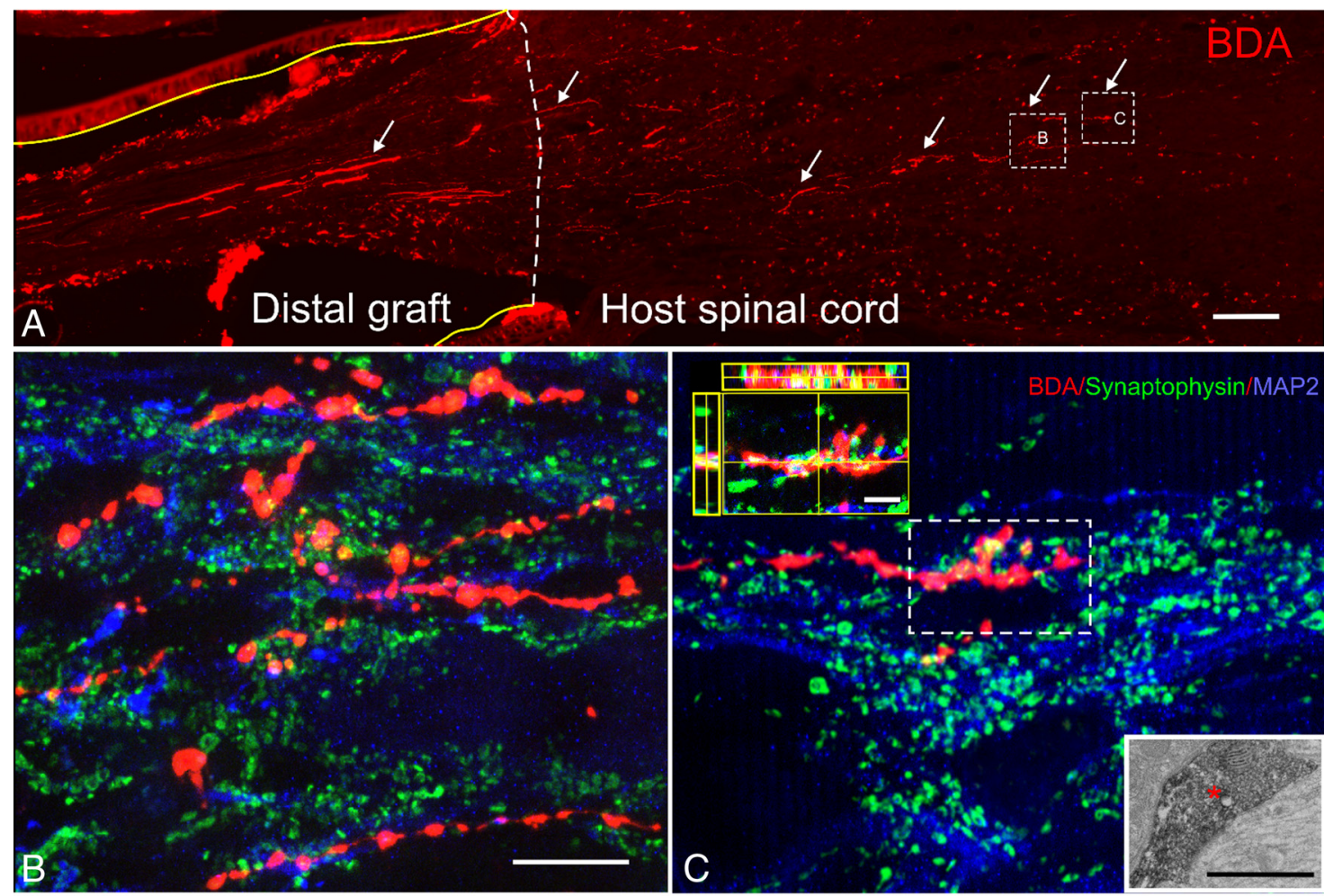

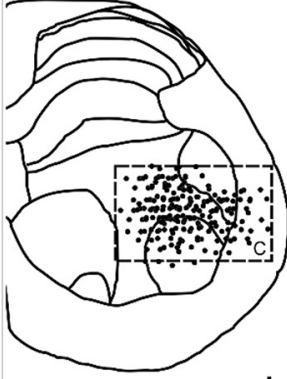

D
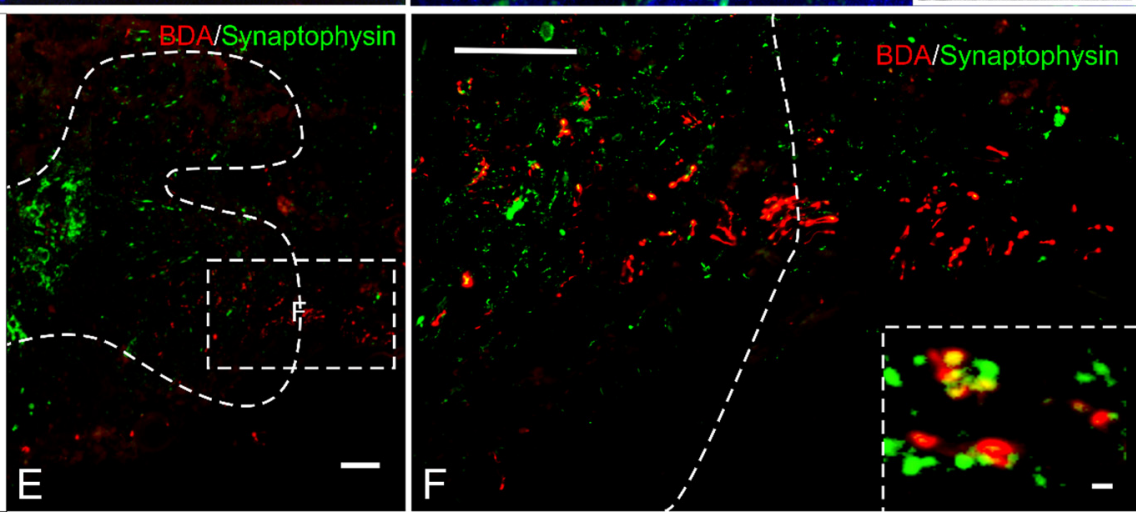

Figure 5. Regenerated descending propriospinal axons formed new synapses on neurons/dendrites within the distal host spinal cord. $\boldsymbol{A}$, BDA-anterogradely labeled descending propriospinal axons (red, arrows) grew through the distal graft-host interface (dashed line) back into the host spinal cord. The grafted guidance channel is outlined by yellow lines. $\boldsymbol{B}$, $\boldsymbol{C}$, High magnification of demarcated areas in $\boldsymbol{A}$ shows sites of new synapse formation (synaptophysin-IR, green) between regenerated axons (BDA-labeled, red) and host dendrites (MAP2-IR; blue). Synapse formation can be further appreciated in the upper left insert in $\boldsymbol{C}$, which shows a high magnification of the boxed area in the same image in single section depicting the relationship between a regenerated axon and a few synaptic terminals on dendrites in $X Y, X Z$, and $Y Z$ planes. The bottom right insert in $C$ is an EM image showing a BDA-labeled presynaptic terminal (*) containing numerous synaptic vesicles. $D$, Schematic drawing plotted the distribution of regenerated axons (dots) in a cross section of the spinal cord at L1. Note that axons appeared in the gray matter of the intermediate and ventral horn areas as well as in the lateral white matter, although the majority was located in the gray matter. $\boldsymbol{E}$, A low magnification of a representative transverse section of $L 1$ spinal cord on the ipsilateral side of the injury showed that regenerated axons, anterogradely labeled with BDA (red), formed synaptic contacts on the dendritic profiles labeled by anti-synaptophysin (green). White dashed line outlines the gray matter. $\boldsymbol{F}$, High magnification of boxed area in $\boldsymbol{E}$ shows colocalization of regenerated axons (BDA, red) with dendritic profiles (synaptophysin-IR, green), which can be further appreciated in a confocal image in an adjacent section (insert of $\boldsymbol{F}$ ). Scale bars: $\boldsymbol{A}, \boldsymbol{E}, \boldsymbol{F}, 100 \mu \mathrm{m} ; \boldsymbol{B}, \boldsymbol{C}, 50 \mu \mathrm{m}$. EM, $500 \mathrm{~nm}$.

then grafted these cells into the distal host spinal cord in an extra group of rats $(n=7)$. We found that grafted SCs-GFP/GDNF survived in all rats (Fig. 6). The average distance of grafted SCs extended from the caudal graft-host interface is $3.14 \pm 1.07 \mathrm{~mm}$. In the caudal spinal cord close to the graft, many BDA-labeled regenerated axons appeared to grow within the SCs-GFP/GDNF territory, whereas others did not (Fig. 6D, G). In the spinal cord farther from the graft, however, the BDA-labeled regenerated axons only appeared to grow within the GFP-SCs territory (Fig. $6 J)$. These results indicated that regenerating axons prefer to grow along with grafted SCs overexpressing GDNF and that they grow faster when being associated with SCs. A subset of regener- ating axons was found to grow outside the SCs territory possibly due to the local GDNF gradient established by grafted SCs overexpressing GDNF. Overall, the grafted SCs overexpressing GDNF are very important in guiding axonal regeneration.

Myelination of axons is a prerequisite for the rapid propagation of nerve impulses. To obtain a good functional recovery, regenerated axons must be myelinated. We examined whether remyelination occurred along regenerated propriospinal axons. The BDA-labeled regenerated axons were found to be myelinated by SCs (Fig. 7A-C). However this myelin could come from either endogenous SCs or grafted SCs. To further determine the source of myelin, we injected SCs-GFP/GDNF, described above, into the 


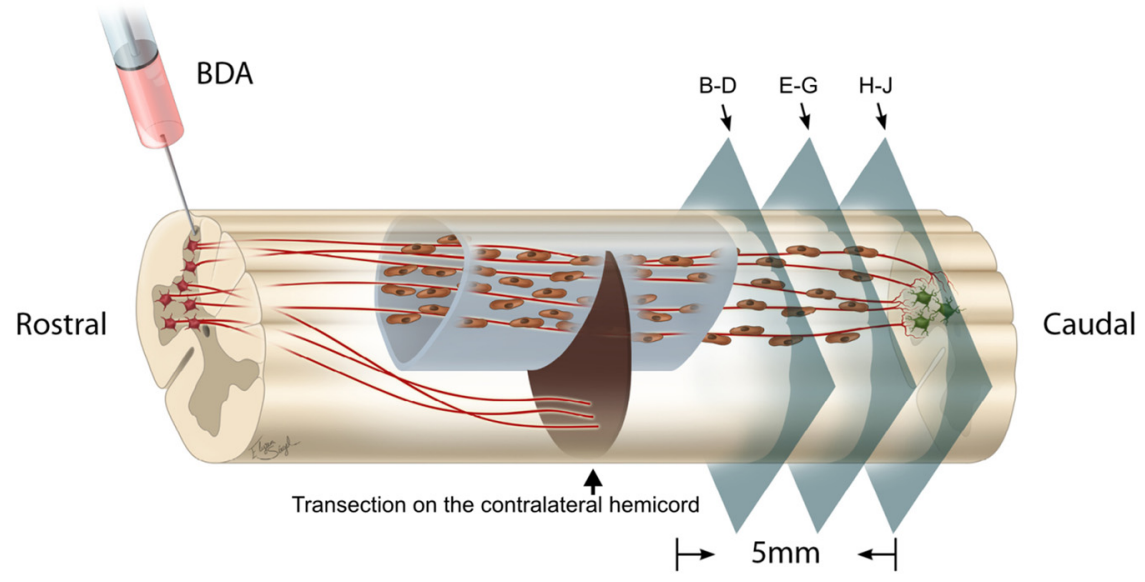

A

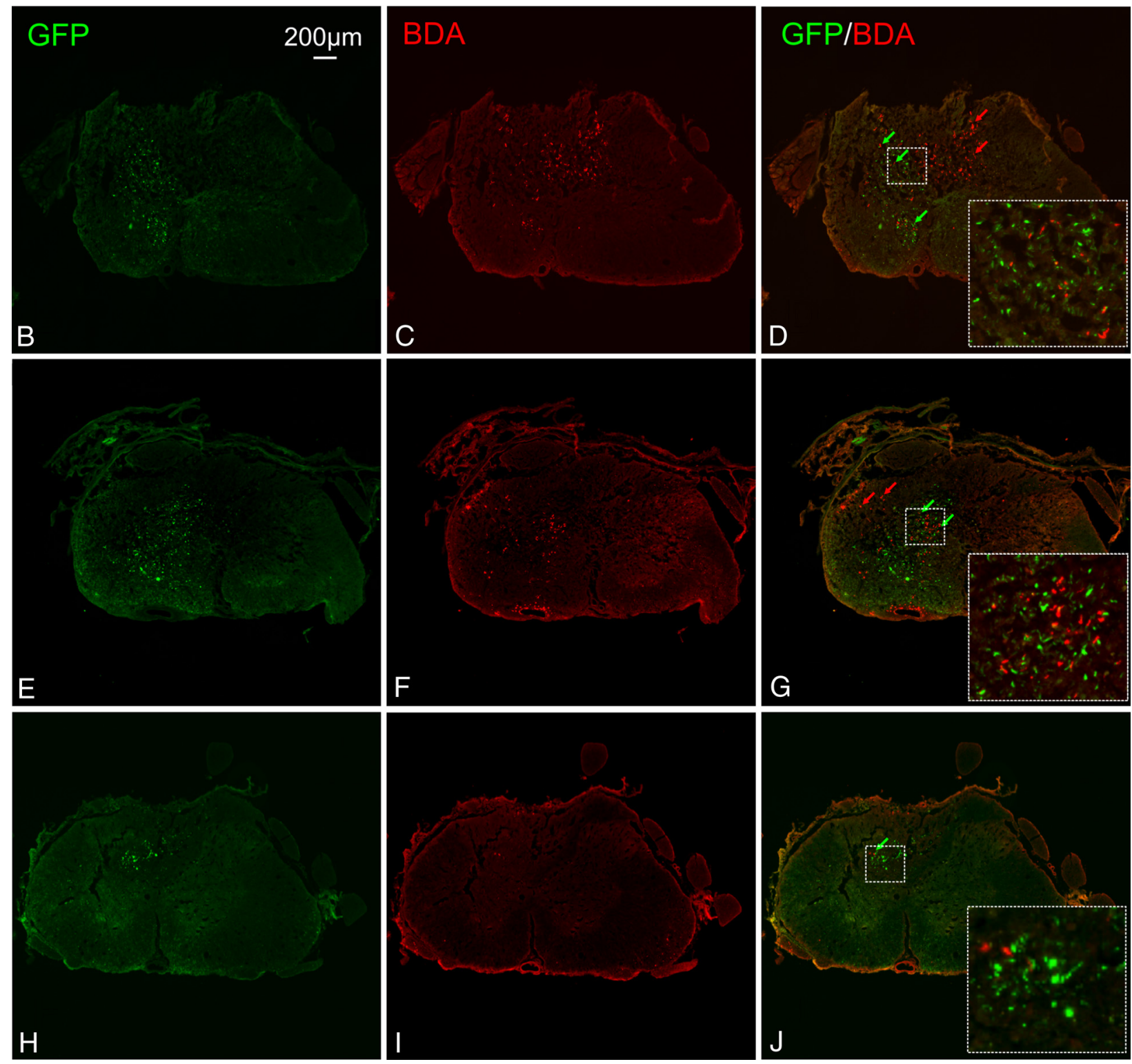

Figure 6. Grafted SCS survived and facilitated axonal regeneration. $A$, Schematic drawing shows how tissue was sampled. Three cross sections caudal to the graft were cut sequentially in rostrocaudal orientation. $\boldsymbol{B}-\boldsymbol{J}$, Representative sections, correspondence to the sections illustrated in $\boldsymbol{A}$, at distances proximal $(\boldsymbol{B}-\boldsymbol{D})$, further $(\boldsymbol{E}-\boldsymbol{G})$, and furthest $(\boldsymbol{H}-\boldsymbol{J})$ to the grafted channel. In the proximal and further sections, BDA-labeled regenerated axons (red) were present not only within the territory of GFP-SCs (green arrows in $\boldsymbol{D}$ and $\boldsymbol{G}$ ) but also in the territory without GFP-SCs (red arrows in $\boldsymbol{D}$ and $\mathbf{G}$ ). In the furthest section, BDA-labeled regenerated axons (red) only appeared in the territory of grafted GFP-SCS (green arrow in $\mathcal{J}$ ). The close association between GFP-SCs and BDA-labeled regenerated axons could be further appreciated in the high-magnification inserts of box areas shown in $\mathbf{D}, \boldsymbol{G}$, and $\boldsymbol{J}$. 


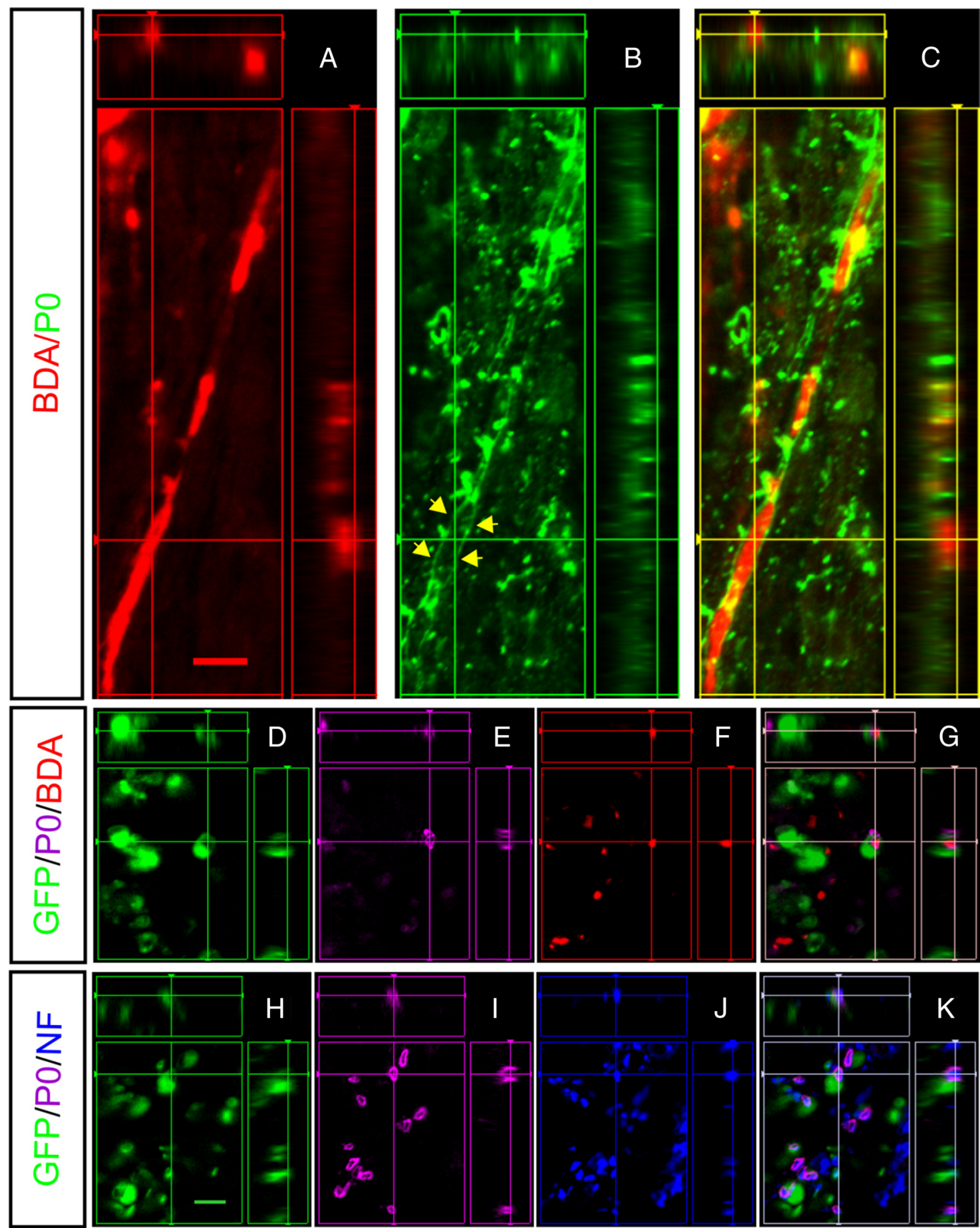

Figure 7. Grafted SCs remyelinated regenerated axons in the distal host spinal cord. $\boldsymbol{A}-\boldsymbol{C}$, At 8 weeks after transplantation, regenerated axons (BDA labeled, red) were myelinated by SCs (P0; green, yellow arrows). $\boldsymbol{D}-\boldsymbol{G}$, Triple labeling of a cross section showed that BDA-labeled regenerated axons (red, $\boldsymbol{F}$ ) were myelinated by grafted SCs (GFP, $\boldsymbol{D})$ that expressed a peripheral myelin marker P0 (purple, $\boldsymbol{E}$ ), which can be further appreciated in the merged image (G). $\boldsymbol{H}-\boldsymbol{K}$, Many grafted SCs (GFP; $\boldsymbol{H})$ formed myelin (P0; purple) on host axons (NF, blue). Scale bar, $10 \mu \mathrm{m}$.

spinal cord caudal to the bridge transplant. We found that grafted SCs-GFP/GDNF remyelinated either regenerated BDA labeled axons (Fig. $7 D-G$ ) or non-BDA-labeled axons (Fig. $7 H-K$ ). Collectively, these results indicate that regenerated axons within the host spinal cord can be myelinated by the grafted or host-derived SCs. It is likely that GDNF played an important role in enhancing SC myelination on regenerated axons, as we demonstrated previously (Zhang et al., 2009).

\section{Regenerated propriospinal axons conducted}

electrophysiological signals across the bridge transplant

To test whether regenerated axons were functional, we examined field potentials in the caudal spinal cord ipsilateral to the injury/ transplantation. As is shown in Figure 8, field potentials were consistently evoked in both sham group $(11.50 \pm 7.17 \mathrm{mV}, n=$ 4) (Fig. $8 G$ ) and the group with SCs-GDNF injections into the caudal cord before contralateral hemisection $(15.13 \pm 5.10 \mathrm{mV}$, 
$n=6, p>0.05$ compared with the sham group) (Fig. $8 A, B, G$ ). In the SCs-GDNFtreated group, the field potentials were reduced by about $45 \%$ after a contralateral hemisection ( $8.68 \pm 2.77 \mathrm{mV}, n=6$ ) (Fig. $8 C, D, G)$, and were almost completely abolished by a subsequent transection of the bridge transplant at its rostral grafthost interface $(1.10 \pm 0.52 \mathrm{mV}, n=3, p<$ 0.05 , compared with prelesion conditions) (Fig. $8 E-G$ ). Contrarily, in the control groups with injections of either SC-GFP or DMEM into the caudal spinal cord, voltage responses were significantly smaller than those in the SCs-GDNF group (SC-GFP: $0.69 \pm 0.11 \mathrm{mV}, n=4$; DMEM: $1.32 \pm 0.77 \mathrm{mV}, n=4)$. Statistically significant differences were found between these two control groups compared with the SCs-GDNF-treated group $(p<0.05$; Fig. 8G).

\section{Recovery of locomotor function}

Last, we examined whether regeneration of propriospinal axons through and beyond the SCs bridging transplant led to recovery of hindlimb locomotor function. Among the three groups, injections of SCs-GDNF into the caudal host spinal cord showed the best improvement for ipsilateral hindlimb function compared with the groups that received either SCsGFP or DMEM injections (Fig. 9A). These animals showed significant differences from controls in gross motor behavior starting between week 4 (BBB scores: DMEM group, $8.42 \pm 1.27, n=5$; SCsGFP group, $8.57 \pm 1.13, n=7$; SCsGDNF group, $10.14 \pm 1.07, n=7$; $p<0.01$ ) and week 6 (BBB scores: DMEM group, $9.14 \pm 0.69, n=5$; SCs-GFP group, $9.14 \pm 0.90, n=7$; SCs-GDNF group, $10.71 \pm 1.25, n=7 ; p<0.05)$. When the intact contralateral spinal cords were transected at week 7 , the right hindlimb function dropped to the lowest level in all groups, possibly due to spinal shock. One week after this second lesion, significant hindlimb function was observed only in the group that received SCs-GDNF injections into the caudal host spinal cord (BBB scores: DMEM group, $0.71 \pm 0.75$, $n=5$; SCs-GFP group, $0.43 \pm 0.79, n=7$; SCs-GDNF group, $5.85 \pm 1.07, n=7 ; p<0.001)$. We also performed footprint analysis with these animals before cutting the contralateral cord. In this analysis, rats with $\mathrm{BBB}$ scores $<9$ were excluded. We chose BBB scores $=9$ as a cutoff to rule out the unqualified rats and to reduce false negative results, because rats with a BBB score $<9$ fail to display either dorsal or plantar stepping. For the right stride length (RSL) in the footprint analysis, we measured the distance between the central points of the imprints, since for rats just reaching the score of 9 , some displayed dorsal stepping, which did not allow the identification of contralateral hemisection.
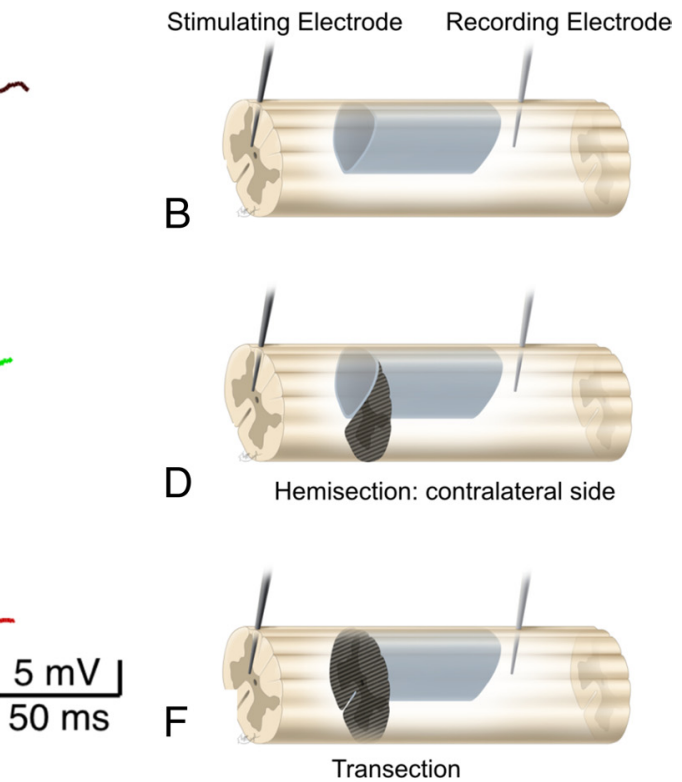
lating electrode located within the spinal intermediate gray region of the rostral spinal cord, and field potentials were recorded in the caudal spinal cord (across the bridge transplant) on the ipsilateral side. Representative traces $(\boldsymbol{A}, \boldsymbol{C}, \boldsymbol{E})$ and related schematic drawings $(\boldsymbol{B}, \boldsymbol{D}, \boldsymbol{F})$ are demonstrated from the same rat that received SCS-GDNF bridge transplant followed by injections of SCS-GDNF gradient into the ipsilateral distal host spinal cord. $\boldsymbol{A}, \boldsymbol{B}$, Field potential was recorded with the contralateral hemicord remained intact. $\boldsymbol{C}, \boldsymbol{D}$, Field potential was recorded after the transection of the contralateral hemicord. $\boldsymbol{E}, \boldsymbol{F}$, Field potential was recorded after the transection of the bridge transplant at the rostral graft-host interface. Note that the signal was abolished after total transection. G, Quantitative data show the amplitude of maximal voltage response in four groups. SC-GDNF treatment group, at both before or after contralateral hemisection, produced a significant increase in amplitude $\left({ }^{*} p<0.05\right)$ when compared with that of either vehicle or SC-GFP treatment group. Importantly, the SC-GDNF treatment-induced increase in amplitude could be almost completely abolished by the total transection $\left({ }^{\#} p<0.05\right)$ compared with the amplitude recorded before and after

their central footpads. Significant differences were found in the RSL (DMEM group, $5.375 \pm 0.7544, n=4$; SCs-GFP group, $6.150 \pm 1.546, n=4$; SCs-GDNF group, $14.21 \pm 1.845, n=7$; $p<0.001)$ between the SCs-GDNF group and the control groups (Fig. 9B).

\section{Discussion}

Here we demonstrate evidence that a continuous growthpromoting pathway can be constructed in the adult rat spinal cord by grafting SCs-GDNF within an SCI and caudal to it, and 

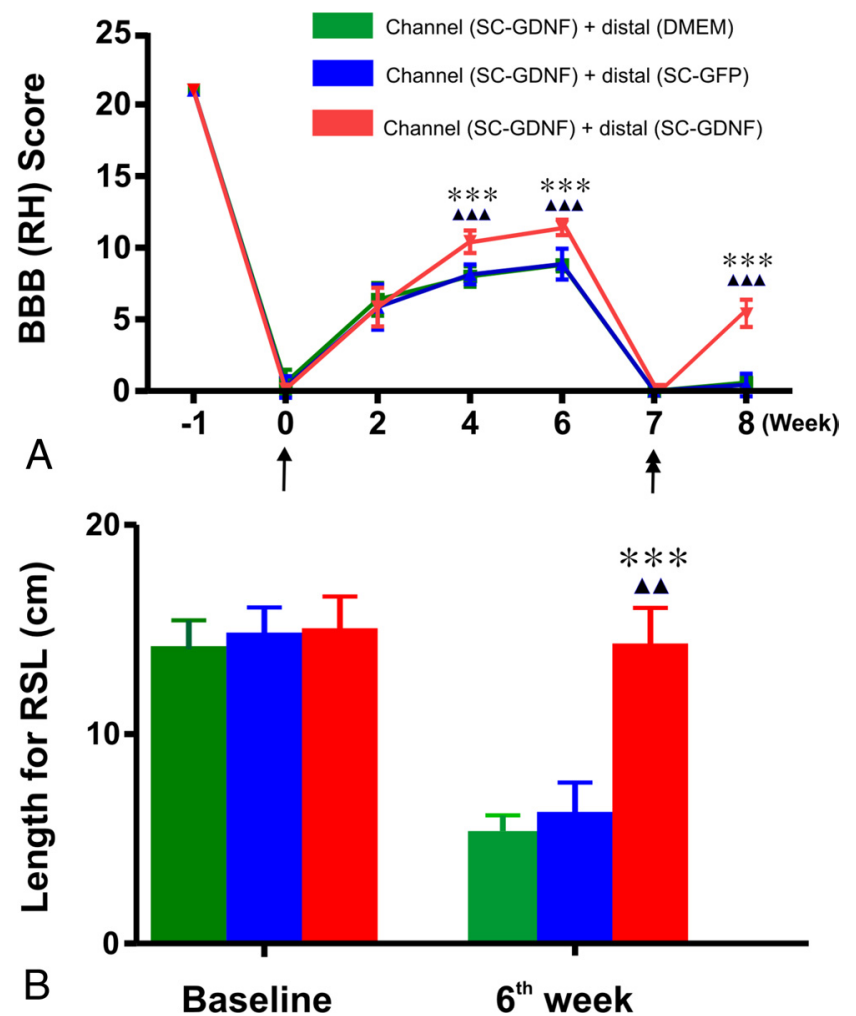

Figure 9. Partial recovery of function after axonal regeneration through a continuous $\mathrm{SCS}$ GDNF growth-promoting pathway. $A$, Improved BBB locomotor recovery was found in the group that received caudal injections of SCs-GDNF (red), compared with caudal injections of SCs-GFP (blue) or DMEM (green) groups ${ }^{* * *} p<0.001$, SCs-GDNF vs SCs-GFP; ${ }^{\mathbf{A \Lambda}} p<0.001$, SCs-GDNF vs DMEM group). The effect of recovery was diminished after contralateral hemisection at week 7 and was partially recovered only in the SCS-GDNF-injected group at week 8. B, Increased stride length on the graft side was found in the group that received caudal injections of SCS-GDNF (red bar) when compared with caudal injections of SCS-GFP (blue bar, ${ }^{* * *} p<$ 0.001 ) or DMEM (green bar, $\left.{ }^{\Delta} \boldsymbol{\Delta}_{p}<0.01\right)$. RSL, right stride length.

that this pathway leads to anatomical regeneration of damaged propriospinal axons from their cut ends to caudal targets of innervation, and formation of new synapses and myelin thereafter. Moreover, such regeneration resulted in partial electrophysiological and behavioral recovery. Given that regeneration of supraspinal axons is limited in our system, it is likely that the supraspinal motor system takes advantage of regenerated propriospinal axons by using this newly formed circuitry as a functional relay to transmit supraspinal commands down to the spinal cord. This repair strategy is encouraged by recent observations that spared long propriospinal axons can form a new intraspinal circuit that relays cortical input to its original spinal targets (Bareyre et al., 2004), and that propriospinal relay connections that bypass one or more injury sites are able to mediate spontaneous recovery and supraspinal control of stepping (Courtine et al., 2008).

Although we used the term regeneration to define the growth of cut axons, some uninjured propriospinal neurons in the vicinity of the lesion might also contribute to the overall axonal growth through and beyond the bridge transplant. Our hemisection model has a limitation in distinguishing between the two. Regardless of regeneration or sprouting, however, these axons grew through and beyond the injury and contributed to functional recovery. To date, no report has shown that sprouting axons have the same growth capacity as regenerating axons.

Although SCs are considered as a strong candidate for transplantation in SCI (Xu et al., 1995; Fouad et al., 2005; Xu and
Onifer, 2009), their migratory ability is impeded by the presence of reactive astrocytes surrounding them (Lakatos et al., 2000; Fairless et al., 2005; Fouad et al., 2005). Moreover, reactive astrocytes increase expression of inhibitory chondroitin sulfate proteoglycans, which form a chemical barrier that imposes major restrictions on axonal growth. To overcome such a growthrestriction at the graft-host interface, we developed this new strategy by providing a continuous growth-permissive pathway, formed by grafted SCs, not only through the lesion gap but also extending to the distal host spinal cord to allow more propriospinal axons to regenerate through and beyond the injury.

To enhance axonal growth through and beyond the injury, SCs were infected to overexpress GDNF. Our previous work showed that GDNF promoted abundant axonal growth within the SCs bridging transplant (Iannotti et al., 2003; Zhang et al., 2009). There are several benefits for the combined use of SCs and GDNF. First, the graft-host interface can be modified to be more permissive to axonal growth (Deng et al., 2011). Second, high concentrations of trophic factor gradient beyond the distal interface may give additional incentive for more axons to leave the graft (Bamber et al., 2001; Tuszynski et al., 2003). Third, many local spinal neurons within motor circuit and glial cells can be rescued by GDNF during the acute phase of the injury (Oppenheim et al., 1995). Last, new synapse formation between regenerated axons and caudal neurons may be enhanced by GDNF (Ledda et al., 2007). Using this novel strategy, numerous axons regenerated back into the caudal host spinal cord, innervating spinal neurons that may influence the activation of the locomotor central pattern generator or ventral motor neurons to execute motor commands. Importantly, regenerated axons displayed good physiological characteristics supporting action potential conduction as well as partial recovery of behavior function.

It is likely that different CNS tracts have different endogenous abilities to regenerate (Richardson et al., 1984). Several supraspinal pathways such as the CST, rubrospinal tract, and descending serotonergic axons, as well as propriospinal axons, could contribute to behavior recovery in different injury models. In our study, by FG retrograde tracing caudal to the injury, we did not detect FG-labeled neurons within supraspinal motor neuron pools. The majority of FG-labeled neurons were confined to propriospinal neurons located in the T8-T10 spinal cord segments. With SCsGDNF treatment, the regeneration of these propriospinal axons through and beyond the transplant was likely due to their expression of the GDNF receptors GFR- $\alpha$ and c-Ret as well as their superior endogenous growth ability compare with other classes of neurons. Since more neurons were retrogradely labeled closer to the injury site, the distance between the site of axotomy and the affected neuronal soma seems to be crucial for determining axonal regeneration. This may be explained by higher expression of growth-associated proteins, such as GAP-43, and cytoskeletal proteins in axons when they were injured closer to the cell body (Doster et al., 1991; Fernandes et al., 1999).

Once regenerated axons cross the distal graft-host interface, they are likely to encounter either the gray or white matter. Both regions have been shown to be growth permissive (Davies et al., 1999; Pettigrew and Crutcher, 1999). To attain more functional recovery, it is necessary to guide the regenerated axons to the specific area in close proximity with host interneurons or motoneurons, which appear to play critical roles in plasticity and recovery of function (Bareyre et al., 2004; Courtine et al., 2008). Although our SCs-GDNF was injected into the intermediate gray matter, regenerated axons appeared in both the white and gray 
matter (Figs. 5D-F, $6 D-G$ ). However, all regenerated axons eventually end within the gray matter where labeled terminal boutonlike structures were observed (Fig. $5 B, C$ ).

A key step toward restoration of function is to promote new synapse and myelin formation after successful regeneration distal to the injury. In our case, we found that axons regenerated in the target area formed new synapses with target neurons/dendrites (Fig. $5 C$, insert). At the ultrastructural level, BDA-labeled presynaptic terminals were clearly seen to contain numerous synaptic vesicles (Fig. 5C, insert). Furthermore, myelination of regenerated axons was found (Fig. 7), suggesting that the regenerated axons could conduct action potentials.

Evidence for functional regeneration in our model was provided by the observation that regenerated axons conducted action potential and animals recover some behavior function when a continuous growth-promoting pathway of SCs-GDNF was provided (Figs. 8, 9). The rostral spinal stimulation in the intermediate gray matter followed by a caudal field potential recording suggested that the regenerated descending propriospinal axons across the lesion, activated the intraspinal circuits below the lesion, and contributed to the majority of functional recovery. However, for the lateral hemisection model, other mechanisms may contribute to recovery as well. One possibility is that transmidline sprouting of descending axons contralateral to the hemisection may provide a potential route for conveying descending axonal input to neuron pools at and below the level of the hemisection (Kerschensteiner et al., 2004; Vavrek et al., 2006; Courtine et al., 2008). Other possibilities are that prelesioned axons from propriospinal neurons project to both the ipsilateral and contralateral caudal spinal cord (Alstermark et al., 1987) or they sprout across the midline after injury (Fenrich and Rose, 2009). It seems that all these potential mechanisms, if they contribute to recovery, should take advantage of the intact, unlesioned contralateral cord. Therefore, to clarify the contribution from propriospinal axons, which truly regenerate through grafted bridges, in both electrophysiological and behavior evaluations, we transected the contralateral intact cord, and observed that the field potentials and behavior recovery remained, although to a lesser degree. Furthermore, relesioning the transplant at the rostral graft-host interface ablated the remaining signals, thus confirming that regenerated axons grew through the bridge transplant and conducted motor signals across the injury rather than bypassing it through the once intact hemicord. Interestingly, with the footprint analysis, we could only find significant differences in RSL between the SCs-GDNF-treated group and the other treatment groups, indicating that functional restoration occurs in more proximal than distal muscles, which could be innervated by different spinal pathways.

Although axonal regeneration, remyelination, and synaptic formation in our case appear to be functional, it remains unclear how efficient these connections were in transducing information. It is possible that the regenerated axons remained in a pathological state with decreased conduction velocities even after regeneration, possibly caused by chronic demyelination (Tan et al., 2007). Although we show evidence that regenerated axons are myelinated, we do not know the extent along the axons or whether the thickness of the myelin surrounding the axons approaches normal. Thus, it is conceivable that, although regenerated axons reformed functional synapses on host neurons, the efficiency of these connections might not be optimal, which may also explain why only limited functional recovery was observed.

In summary, we have demonstrated that a growth-promoting pathway, formed by grafted SCs-GDNF, promoted a continuous regeneration of DPST axons through and beyond the lesion gap of a spinal cord hemisection. Within the distal host spinal cord, regenerated DPST axons formed new synapses on host neurons leading to partial restoration of action potentials and behavioral functions. Although our strategy is still experimental, it represents a proof of principle of a novel treatment opportunity, which is especially important considering the large number of patients presenting with severe spinal cord injuries.

\section{References}

Abdellatif AA, Pelt JL, Benton RL, Howard RM, Tsoulfas P, Ping P, Xu XM, Whittemore SR (2006) Gene delivery to the spinal cord: comparison between lentiviral, adenoviral, and retroviral vector delivery systems. J Neurosci Res 84:553-567. CrossRef Medline

Alstermark B, Kümmel H, Pinter MJ, Tantisira B (1987) Branching and termination of $\mathrm{C} 3-\mathrm{C} 4$ propriospinal neurones in the cervical spinal cord of the cat. Neurosci Lett 74:291-296. CrossRef Medline

Alstermark B, Isa T, Tantisira B (1991) Integration in descending motor pathways controlling the forelimb in the cat. 18. Morphology, axonal projection and termination of collaterals from C3-C4 propriospinal neurones in the segment of origin. Exp Brain Res 84:561-568. Medline

Bamber NI, Li H, Lu X, Oudega M, Aebischer P, Xu XM (2001) Neurotrophins BDNF and NT-3 promote axonal re-entry into the distal host spinal cord through Schwann cell-seeded mini-channels. Eur J Neurosci 13:257268. CrossRef Medline

Bareyre FM, Kerschensteiner M, Raineteau O, Mettenleiter TC, Weinmann O, Schwab ME (2004) The injured spinal cord spontaneously forms a new intraspinal circuit in adult rats. Nat Neurosci 7:269-277. CrossRef Medline

Basso DM, Beattie MS, Bresnahan JC (1995) A sensitive and reliable locomotor rating scale for open field testing in rats. J Neurotrauma 12:1-21. CrossRef Medline

Bunge MB (2008) Novel combination strategies to repair the injured mammalian spinal cord. J Spinal Cord Med 31:262-269. Medline

Campos L, Meng Z, Hu G, Chiu DT, Ambron RT, Martin JH (2004) Engineering novel spinal circuits to promote recovery after spinal injury. J Neurosci 24:2090-2101. CrossRef Medline

Conta AC, Stelzner DJ (2004) Differential vulnerability of propriospinal tract neurons to spinal cord contusion injury. J Comp Neurol 479:347359. CrossRef Medline

Courtine G, Song B, Roy RR, Zhong H, Herrmann JE, Ao Y, Qi J, Edgerton VR, Sofroniew MV (2008) Recovery of supraspinal control of stepping via indirect propriospinal relay connections after spinal cord injury. Nat Med 14:69-74. CrossRef Medline

David S, Aguayo AJ (1981) Axonal elongation into peripheral nervous system "bridges" after central nervous system injury in adult rats. Science 214:931-933. CrossRef Medline

Davies SJ, Goucher DR, Doller C, Silver J (1999) Robust regeneration of adult sensory axons in degenerating white matter of the adult rat spinal cord. J Neurosci 19:5810-5822. Medline

Deng LX, Hu J, Liu N, Wang X, Smith GM, Wen X, Xu XM (2011) GDNF modifies reactive astrogliosis allowing robust axonal regeneration through Schwann cell-seeded guidance channels after spinal cord injury. Exp Neurol 229:238-250. CrossRef Medline

Doster SK, Lozano AM, Aguayo AJ, Willard MB (1991) Expression of the growth-associated protein GAP-43 in adult rat retinal ganglion cells following axon injury. Neuron 6:635-647. CrossRef Medline

Fairless R, Frame MC, Barnett SC (2005) N-cadherin differentially determines Schwann cell and olfactory ensheathing cell adhesion and migration responses upon contact with astrocytes. Mol Cell Neurosci 28:253-263. CrossRef Medline

Fenrich KK, Rose PK (2009) Spinal interneuron axons spontaneously regenerate after spinal cord injury in the adult feline. J Neurosci 29:1214512158. CrossRef Medline

Fernandes KJ, Fan DP, Tsui BJ, Cassar SL, Tetzlaff W (1999) Influence of the axotomy to cell body distance in rat rubrospinal and spinal motoneurons: differential regulation of GAP-43, tubulins, and neurofilament-M. J Comp Neurol 414:495-510. CrossRef Medline

Fouad K, Schnell L, Bunge MB, Schwab ME, Liebscher T, Pearse DD (2005) Combining Schwann cell bridges and olfactory-ensheathing glia grafts 
with chondroitinase promotes locomotor recovery after complete transection of the spinal cord. J Neurosci 25:1169-1178. CrossRef Medline

Houle JD, Tom VJ, Mayes D, Wagoner G, Phillips N, Silver J (2006) Combining an autologous peripheral nervous system "bridge" and matrix modification by chondroitinase allows robust, functional regeneration beyond a hemisection lesion of the adult rat spinal cord. J Neurosci 26: 7405-7415. CrossRef Medline

Iannotti C, Li H, Yan P, Lu X, Wirthlin L, Xu XM (2003) Glial cell linederived neurotrophic factor-enriched bridging transplants promote propriospinal axonal regeneration and enhance myelination after spinal cord injury. Exp Neurol 183:379-393. CrossRef Medline

Illert M, Lundberg A, Tanaka R (1977) Integration in descending motor pathways controlling the forelimb in the cat. 3. Convergence on propriospinal neurones transmitting disynaptic excitation from the corticospinal tract and other descending tracts. Exp Brain Res 29:323-346. Medline

Kerschensteiner M, Bareyre FM, Buddeberg BS, Merkler D, Stadelmann C, Brück W, Misgeld T, Schwab ME (2004) Remodeling of axonal connections contributes to recovery in an animal model of multiple sclerosis. J Exp Med 200:1027-1038. CrossRef Medline

Lakatos A, Franklin RJ, Barnett SC (2000) Olfactory ensheathing cells and Schwann cells differ in their in vitro interactions with astrocytes. Glia 32:214-225. CrossRef Medline

Ledda F, Paratcha G, Sandoval-Guzmán T, Ibáñez CF (2007) GDNF and GFRalpha1 promote formation of neuronal synapses by ligand-induced cell adhesion. Nat Neurosci 10:293-300. CrossRef Medline

Liu Y, Wang X, Lu CC, Kerman R, Steward O, Xu XM, Zou Y (2008) Repulsive Wnt signaling inhibits axon regeneration after CNS injury. J Neurosci 28:8376-8382. CrossRef Medline

Matsushita M, Ikeda M, Hosoya Y (1979) The location of spinal neurons with long descending axons (long descending propriospinal tract neurons) in the cat: a study with the horseradish peroxidase technique. J Comp Neurol 184:63-80. CrossRef Medline

Molenaar I, Kuypers HG (1978) Cells of origin of propriospinal fibers and of fibers ascending to supraspinal levels. A HRP study in cat and rhesus monkey. Brain Res 152:429-450. CrossRef Medline

Morrissey TK, Kleitman N, Bunge RP (1991) Isolation and functional characterization of Schwann cells derived from adult peripheral nerve. J Neurosci 11:2433-2442. Medline

Oppenheim RW, Houenou LJ, Johnson JE, Lin LF, Li L, Lo AC, Newsome AL, Prevette DM, Wang S (1995) Developing motor neurons rescued from programmed and axotomy-induced cell death by GDNF. Nature 373: 344-346. CrossRef Medline

Perrelet D, Ferri A, Liston P, Muzzin P, Korneluk RG, Kato AC (2002) IAPs are essential for GDNF-mediated neuroprotective effects in injured motor neurons in vivo. Nat Cell Biol 4:175-179. CrossRef Medline

Pettigrew DB, Crutcher KA (1999) White matter of the CNS supports or inhibits neurite outgrowth in vitro depending on geometry. J Neurosci 19:8358-8366. Medline
Reed WR, Shum-Siu A, Whelan A, Onifer SM, Magnuson DS (2009) Anterograde labeling of ventrolateral funiculus pathways with spinal enlargement connections in the adult rat spinal cord. Brain Res 1302:76-84. CrossRef Medline

Richardson PM, McGuinness UM, Aguayo AJ (1980) Axons from CNS neurons regenerate into PNS grafts. Nature 284:264-265. CrossRef Medline

Richardson PM, Issa VM, Aguayo AJ (1984) Regeneration of long spinal axons in the rat. J Neurocytol 13:165-182. CrossRef Medline

Sivasankaran R, Pei J, Teng YD, Wang KC, Zhang YP, Shields CB, Xu XM, He Z (2004) PKC mediates inhibitory effects of myelin and chondroitin sulfate proteoglycans on axonal regeneration. Nat Neurosci 7:261-268. CrossRef Medline

Tan AM, Petruska JC, Mendell LM, Levine JM (2007) Sensory afferents regenerated into dorsal columns after spinal cord injury remain in a chronic pathophysiological state. Exp Neurol 206:257-268. CrossRef Medline

Titsworth WL, Onifer SM, Liu NK, Xu XM (2007) Focal phospholipases A2 group III injections induce cervical white matter injury and functional deficits with delayed recovery concomitant with Schwann cell remyelination. Exp Neurol 207:150-162. CrossRef Medline

Tuszynski MH, Grill R, Jones LL, Brant A, Blesch A, Löw K, Lacroix S, Lu P (2003) NT-3 gene delivery elicits growth of chronically injured corticospinal axons and modestly improves functional deficits after chronic scar resection. Exp Neurol 181:47-56. CrossRef Medline

Vavrek R, Girgis J, Tetzlaff W, Hiebert GW, Fouad K (2006) BDNF promotes connections of corticospinal neurons onto spared descending interneurons in spinal cord injured rats. Brain 129:1534-1545. CrossRef Medline

Xu XM, Onifer SM (2009) Transplantation-mediated strategies to promote axonal regeneration following spinal cord injury. Respir Physiol Neurobiol 169:171-182. CrossRef Medline

Xu XM, Guénard V, Kleitman N, Bunge MB (1995) Axonal regeneration into Schwann cell-seeded guidance channels grafted into transected adult rat spinal cord. J Comp Neurol 351:145-160. CrossRef Medline

Xu XM, Chen A, Guénard V, Kleitman N, Bunge MB (1997) Bridging Schwann cell transplants promote axonal regeneration from both the rostral and caudal stumps of transected adult rat spinal cord. J Neurocytol 26:1-16. CrossRef Medline

Xu XM, Zhang SX, Li H, Aebischer P, Bunge MB (1999) Regrowth of axons into the distal spinal cord through a Schwann-cell-seeded mini-channel implanted into hemisected adult rat spinal cord. Eur J Neurosci 11:17231740. CrossRef Medline

Yan P, Xu J, Li Q, Chen S, Kim G-M, Hsu CY, Xu XM (1999) Glucocorticoid receptor expression in the spinal cord after traumatic injury in adult rats. J Neurosci 19:9355-9363. Medline

Zhang L, Ma Z, Smith GM, Wen X, Pressman Y, Wood PM, Xu XM (2009) GDNF-enhanced axonal regeneration and myelination following spinal cord injury is mediated by primary effects on neurons. Glia 57:1178 1191. CrossRef Medline 